\title{
Optically controlled entangling gates in randomly doped silicon
}

\author{
Eleanor Crane $\odot,{ }^{1, *}$ Thomas Crane, ${ }^{2}$ Alexander Schuckert $\odot,{ }^{3}$ Nguyen H. Le, ${ }^{4}$ Kristian Stockbridge, ${ }^{4}$ \\ Steven Chick, ${ }^{4}$ and Andrew J. Fisher ${ }^{1,5}$ \\ ${ }^{1}$ London Centre for Nanotechnology, University College London, Gower Street, London WC1E 6BT, United Kingdom \\ ${ }^{2}$ Laboratoire SPHERE, Université Paris Diderot, 5 Rue Thomas Mann, Paris 75013, France \\ ${ }^{3}$ Department of Physics, Technical University of Munich, 85748 Garching, Germany \\ ${ }^{4}$ Advanced Technology Institute and Department of Physics, University of Surrey, Guildford GU2 7XH, United Kingdom \\ ${ }^{5}$ Department of Physics \& Astronomy, University College London, Gower Street, London, WC1E 6BT, United Kingdom
}

(Received 23 March 2019; revised manuscript received 6 June 2019; published 8 August 2019)

\begin{abstract}
Donor qubits in bulk doped silicon have many competitive advantages for quantum computation in the solid state: not only do they offer a fast way to scalability, but they also show some of the longest coherence times found in any quantum computation proposal. We determine the densities of entangling gates in randomly doped silicon comprising two different dopant species. First, we define conditions and plot maps of the relative locations of the dopants necessary for them to form exchange interaction-mediated entangling gates. Second, using nearest neighbor Poisson point process theory, we calculate the doping densities necessary for maximal densities of single and dual-species gates. Third, using the moving-average cluster expansion technique, we make predictions for a proof of principle experiment demonstrating the control of the far-from-equilibrium magnetization dynamics of one species by the orbital excitation of another. We find agreement of our results with a Monte Carlo simulation that handles multiple donor structures and scales optimally with the number of dopants. The simulator can also extract donor structures not captured by our Poisson point process theory. The combined approaches to density optimization in random distributions presented here may be useful for other condensed matter systems as well as applications outside physics.
\end{abstract}

DOI: 10.1103/PhysRevB.100.064201

\section{INTRODUCTION}

Initial successes in the realization of coherent quantum operations on collections of quantum bits (qubits), mainly on AMO platforms such as trapped ions $[1,2]$ and ultracold atoms in optical lattices [3-6], have shown how hard it is to construct a quantum computer large enough to outperform its classical counterparts. This scalability challenge might be more easily overcome in solid-state realizations, for which a wide range of qubits have been proposed, including Majorana fermions in nanowires [7,8], superconducting qubits [9-11], nitrogen-vacancy centers in diamond [12], quantum dots [13], and donor impurities in silicon $[14,15]$. Superior scaling might not only unlock date-based algorithms such as factoring [16], unstructured search [17], and machine learning [18] but may also lead to a better understanding of strongly correlated systems through Feynman's idea of quantum simulation [19-22].

A realization in doped silicon, the most important material in the electronics industry today, could point the way forward for the widespread introduction of quantum computation. Donor impurities have several spin-1/2 degrees of freedom which provide natural Hilbert spaces to encode qubits [23]. The nuclear spins of ${ }^{31} \mathrm{P}[24,25]$ and the electron spin of the valence electron have some of the longest decoherence times of any qubit realizations.

*e.crane@ucl.ac.uk

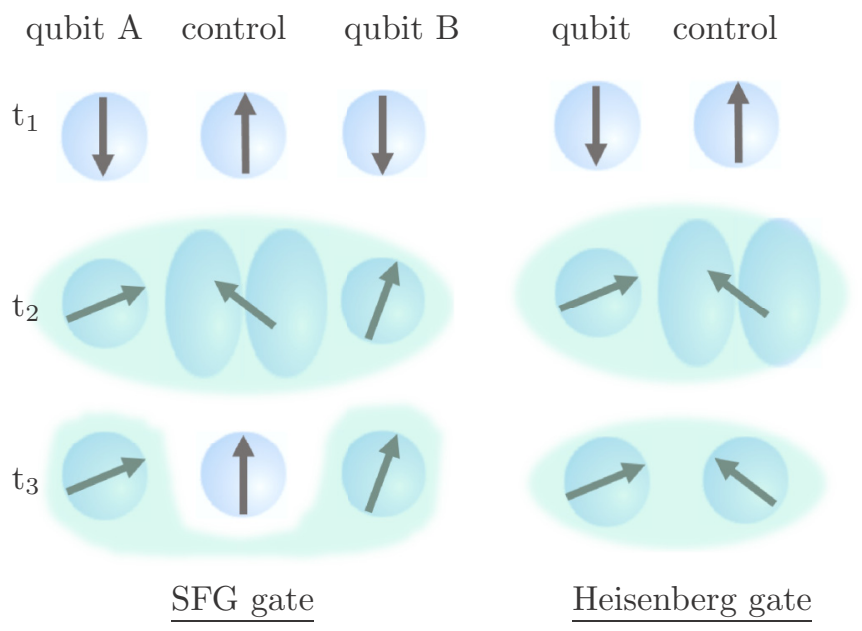

FIG. 1. Optically controlled interaction-exchange-energy entangling gates in donors in silicon. At time $t_{1}$, all donors are noninteracting in their ground state; at time $t_{2}$, the controls are excited to a higher-lying and physically extended orbital state, entangling themselves via Heisenberg exchange with the neighboring qubits of a different donor species; finally, at $t_{3}$, the control donor electron falls back to the ground state. In the Stoneham-Fisher-Greenland (SFG) [26] scheme (left), the excited controls mediate entangling interactions between pairs of qubits and the control is then removed from the entangled state on de-excitation [27] while, in the Heisenberg scheme (right), the entanglement is produced between a qubit and the control. 
These range from hours to several days [28], especially when benefiting from isotopically pure ${ }^{28} \mathrm{Si}$ and/or fieldinsensitive clock transitions [29,30]. Initialization and readout [31] and single-qubit operations have been demonstrated on single electron spins [28] via metallic microwave strip lines [32] and donor-bound exciton spectroscopy [33,34]. However, in addition to initialization, readout, and single-qubit operations, a two-qubit entangling gate is required for universal quantum computation [35].

Several proposals to realize entangling gates in silicon exploit the exchange interactions between ground-state electronic states; these interactions are strongly oscillatory because of interference between the different valley components of donor wave functions [36] and typically require atomicscale precision in the positioning of the donors [24]. This needs specialized lithography based on selective removal of hydrogen atoms by scanning tunneling microscope [37] and subsequent deposition of donor species [38], a delicate and resource-intensive technique $[32,38,39]$. Schemes based on electric-dipole interactions have larger donor spacings and tolerate less stringent fabrication requirements [40]. However, a scheme capable of inducing entangling interactions with truly random placement of donors would allow relatively seamless integration of the fabrication into the standard widespread silicon processing industry. In 2D, the donor locations could be established using scanning tunneling microscopy at the early stages of fabrication, enabling identification of suitable gate geometries.

In this paper, we discuss a family of entangling gate implementations, sketched in Fig. 1, that make use of physically localized or extended orbital excited states to control the interactions between donor qubits in a disordered ensemble [26]. These orbital excited states can be produced by excitation with terahertz radiation [41], with multiple schemes offering the necessary single-qubit addressability. Because donors are randomly positioned, there is inhomogeneous broadening due to differing local magnetic fields generated by residual ${ }^{29} \mathrm{Si}$ nuclear spins. This could enable selective addressing of subensembles with a specific frequency of terahertz radiation [42]. The spread of frequencies could be further increased by placing the sample in a field gradient, so each donor has a different transition frequency [42]. The excited states produced (for example, $2 p$ orbitals) have a spatial structure that depends on the axis of polarization of the laser, thus providing another tool for spatial selectivity within local configurations. Important steps have been made toward the realization of exchange-interaction-based gates in donors in silicon. The disentanglement of the control donor from the two entangled qubits was shown to be feasible after the gate operation [27]. Coherent control of the valence electron orbital states of dopants such as phosphorus, bismuth, antimony, and arsenic has been demonstrated with terahertz light, using a free electron laser (FEL) tuned to the low tens of meV [41]. Furthermore, initialization and readout can be done optically, using donor-bound exciton spectroscopy in the terahertz frequencies, which was demonstrated experimentally with phosphorus spins in silicon [43]. Until now, it has remained unclear what densities of "viable" entangling gates could be reached and even what the requirements are for a configuration to be viable.
In this paper, we define suitable conditions for a viable gate geometry and obtain the maximal gate densities for $2 \mathrm{D}$ or $3 \mathrm{D}$ homogeneous doping. These correspond to the ideal limits of a doped bulk sample or delta layer, respectively; we also consider the case where two parallel ideal delta layers are implanted with different species. The general case of a spatially varying density (hence, allowing both for graded doping of a bulk material and for the inevitable broadening of real delta layers) will be treated in a subsequent paper [44]; in that paper, a heuristic will also be given which enables fast estimates of the densities of viable clusters over a wide density range.

This paper is organized as follows. First, we estimate appropriate conditions on the spacing of the dopants required for them to form a viable entangling gate. Second, optimal doping densities to produce the highest densities of entangling gates are calculated using a Monte Carlo simulation and Poisson point process theory. Third, we propose a proof-of-principle experiment to show control over the exchange interaction between donors at the optimal doping densities and predict its results with the moving-average cluster expansion (MACE) method [45]. Our numerical results apply to the case of group $\mathrm{V}$ donors in silicon; however, our methods apply to any material where particular configurations of multiple impurities are required, and the conditions on the configurations needed can be expressed in terms of distances between different species.

\section{GEOMETRIC CONDITIONS ON OPTICALLY CONTROLLED ENTANGLING GATE CONFIGURATIONS}

First, we define conditions on the distances between dopants to form a Stoneham-Fisher-Greenland (SFG) entangling gate by comparing energy scales corresponding to the lifetime of the excited state [46,47], the strength of the entangling exchange interaction, and the requirement for qubits to be isolated from each other when not part of the entangling operation. These same considerations are also applicable to the Heisenberg entangling gate. Based on these conditions, we then calculate maps and line scans of the interaction exchange energy from a theory including multivalley interference [36] to delimit areas around donors in which entangling gate operation is possible.

\section{A. Conditions on the exchange-interaction energy}

A phase gate (which can be combined with Hadamard gates to result in a controlled NOT gate) [48] can be implemented by the sequence

$$
\hat{u}_{\text {phase }}=\hat{r}_{2}^{z}\left(-\frac{\pi}{2}\right) \hat{r}_{1}^{z}\left(\frac{\pi}{2}\right) \hat{e}_{12}\left(\frac{\pi}{2}\right) \hat{r}_{1}^{z}(\pi) \hat{e}_{12}\left(\frac{\pi}{2}\right),
$$

where the $\hat{r}_{i}^{z}$ are single-qubit operations. The two-qubit operation is given by $\hat{e}_{12}\left(\frac{\pi}{2}\right)=e^{-i \frac{\pi}{2} \hat{H}_{12} / J}$, where $\hat{H}_{12}=J \hat{\mathbf{S}}_{1} \hat{\mathbf{S}}_{2}$ is the Heisenberg Hamiltonian between spins $\mathbf{S}_{1}$ and $\mathbf{S}_{2}$. Equating this with the time evolution operator $\hat{U}=e^{-\frac{i}{\hbar} t \hat{H}_{12}}$ leads to the condition

$$
J=\frac{h}{4 t} \quad \text { or } \quad t=\frac{h}{4 J}
$$

for a successful phase gate operation.

The exchange interaction constant $J$ arises from overlap of spatially separated wave functions. The value of $J$ (or $t$ ) must 
be controlled such that Eq. (2) is fulfilled, where the principle source of imperfect gate operation results from interactions between the gate dopants and other donors. In silicon, it is especially challenging to fulfill this requirement as the exchange interaction between valence dopant electrons not only decays exponentially with distance but also oscillates with a period of the order of the lattice spacing due to intervalley interference [49]. The lifetimes $T_{1}$ of the donor excited states are limited by phonon-mediated decay, and for phosphorus and arsenic they have been measured as $T_{1}=200 \mathrm{ps}$ [46], which limits the gate operation time to $t<T_{1}$; hence, we define an energy scale:

$$
J_{\mathrm{dec}}=\frac{h}{4 T_{1}} \text {. }
$$

Since for proof-of-principle experiments we would require a population of excited-state control spins, but not coherence between the ground-state and excited-state wave packets, Eq. (3) sets the minimum exchange energy for a successful two-qubit gate. The phase coherence time $T_{2}$ is bounded by $2 T_{1}$ and has been measured to be $160 \mathrm{ps}$ in Czochralski grown silicon and is estimated to be above $320 \mathrm{ps}$ in isotopically pure silicon [41].

In the rest of this paper, we will consider two group-V donor species: The shallow donor phosphorus $(\mathrm{Si}: \mathrm{P})$ as the controls (also referred to as $\mathrm{c}$ in the general case), whose excited-state wave functions mediate the interactions between the deeper arsenic (Si:As) ground-state spins as the readout qubits (also referred to as $r$ if not specifically considering an implementation relying on using this particular species).

\section{SFG entangling gate}

As represented in Fig. 1, we assume that only the control donors are excited to a higher-lying orbital state which we will choose to be the $2 p$ excited state; the readout donors remain in the ground state. The following conditions then need to be met for successful gate switching operation:

Readouts. Interaction between readouts should be smaller than the decoherence interaction-exchange energy defined in Eq. (3), i.e., $J\left(\mathrm{r}_{1 s}-\mathrm{r}_{1 s}\right)(r)<J_{\mathrm{dec}}$. We will denote the distance at which these two scales are equal as $R_{r r}$.

Readout control. If the control is in the ground $(1 s)$ state, the interactions between readout and control should also be small: $J\left(\mathrm{c}_{1 s}-\mathrm{r}_{1 s}\right)(r)<J_{\mathrm{dec}}$, with equality at $R_{\min }$. Furthermore, if the control is in the excited state, control and readout should interact, therefore $J\left(\mathrm{c}_{2 p}-\mathrm{r}_{1 s}\right)(r)>J_{\mathrm{dec}}$ with equality at $R_{\max }$.

Controls. If two neighboring controls are excited to the $2 p$, their interaction should not dominate the process, i.e., $J\left(\mathrm{c}_{2 p}-\mathrm{c}_{2 p}\right)(r)<J_{\mathrm{dec}}$ with equality at $R_{c c}$.

The distances introduced above are shown in Fig. 2 in the form of multivalley exchange interactions and disk approximations discussed in the next section.

In the above considerations, we did not specify the number of readouts per control. Although originally devised for two readouts [26], we will leave this number general.

\section{Heisenberg entangling gate}

There are two ways of implementing this gate. In the first, which we refer to as excited-ground Heisenberg gate, one donor is in the excited and the other in the ground state; this configuration is the same as the SFG gate with just one

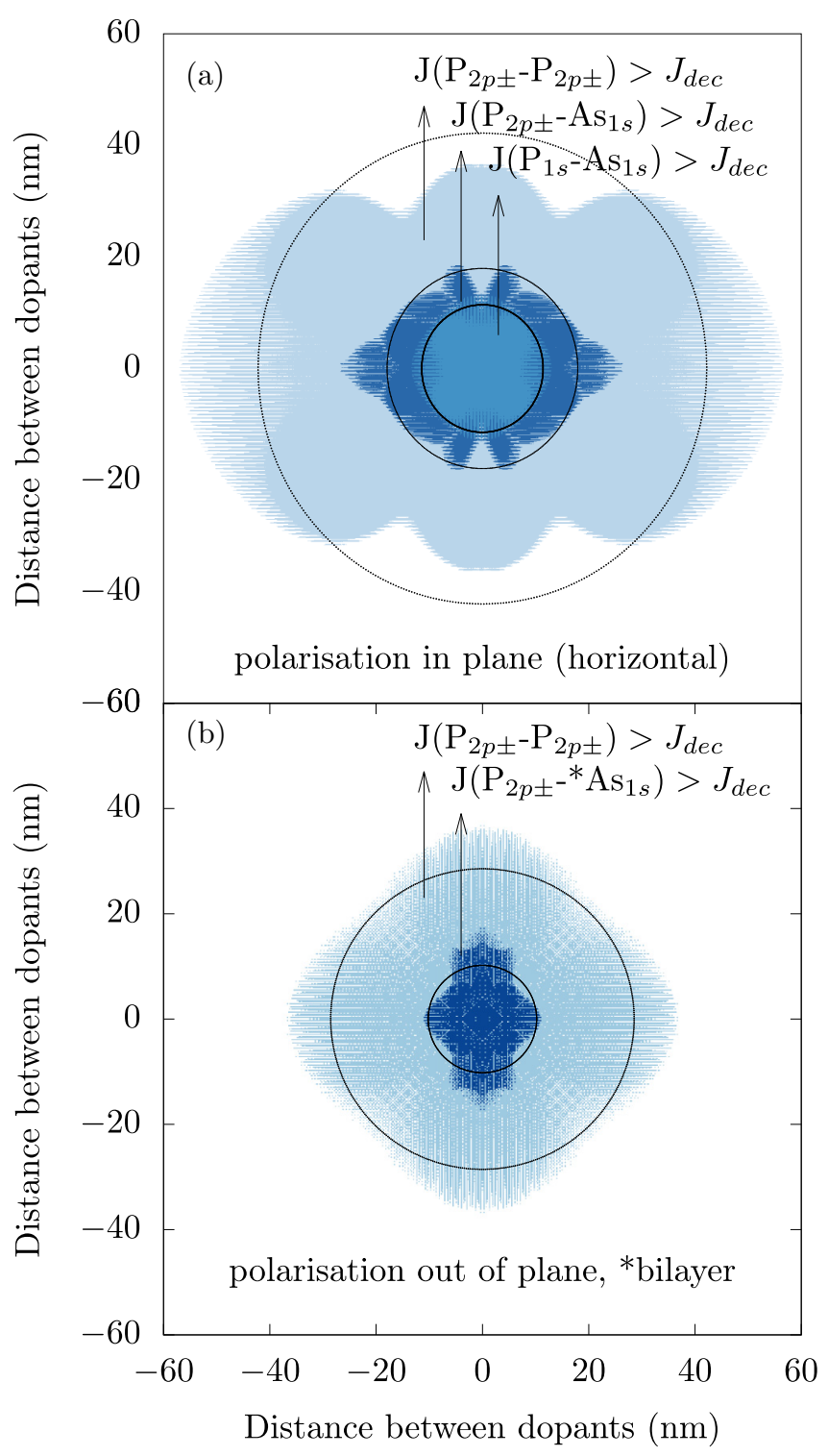

\begin{tabular}{c|c|c|c} 
Polarisation & Interaction & Name at $J_{\text {dec }}$ & Distance (nm) \\
\hline \multirow{6}{*}{$e_{\|}$} & $\mathrm{J}\left(\mathrm{As}_{1 s}-\mathrm{As}_{1 s}\right)$ & $R_{r r}$ & 11.0 \\
& $\mathrm{~J}\left(\mathrm{P}_{1 s}-\mathrm{As}_{1 s}\right)$ & $R_{\text {min }}$ & 11.4 \\
& $\mathrm{~J}\left(\mathrm{P}_{2 p \pm}-\mathrm{As}_{1 s}\right)$ & $R_{\text {max }}$ & 17.9 \\
& $\mathrm{~J}\left(\mathrm{P}_{2 p \pm}-\mathrm{P}_{2 p \pm}\right)$ & $R_{c c}$ & 42.2 \\
& $\mathrm{~J}\left(\mathrm{P}_{1 s}-\mathrm{P}_{1 s}\right)$ & $R_{\min }^{\prime}$ & 11.8 \\
\hline \multirow{2}{*}{$e_{\perp}$} & $\mathrm{J}\left(\mathrm{P}_{2 p 土^{-}} \mathrm{As}_{1 s}\right)$ & $R_{\max } *$ & 10.2 \\
& $\mathrm{~J}\left(\mathrm{P}_{2 p \pm}-\mathrm{P}_{2 p \pm}\right)$ & $R_{c c}$ & 28.5 \\
\hline
\end{tabular}

FIG. 2. Interaction exchange energy (J) limits (a) in the layer in which arsenic and phosphorus are doped and the light is polarized in plane $e_{\|}$and (b) in the layer where arsenic is doped, which is $13.2 \mathrm{~nm}$ away from the phosphorus layer (avoiding all ground-state multivalley interactions within the decay time) and parallel to it, and the light is polarized out of plane $e_{\perp} . *$ indicates the "bilayer" case. In the colored areas, $\mathbf{J}>\mathrm{J}_{\mathrm{dec}}$, defined in Eq. (3). In (a), the horizontal distortion of the exchange involving the $p$ state is due to the linearly polarized light being polarized in plane. Circles represent the discs whose areas are equal to those of the interaction zones, multivalley effects taken into account. The table defines the cutoff distances used in terms of the interaction strengths, and gives the values of the distances used in our numerical results. 
readout. In the second case, the excited-excited Heisenberg gate, both donors are excited. This can be a single-species gate composed of two Si:P electrons, for example.

In the excited-ground case, the conditions are the same as for the SFG gate, except only one readout should interact strongly with a control, and not two.

For the excited-excited Heisenberg gate, the dominant process should be the $2 p \pm$ state of both controls interacting with each other: $\mathrm{J}\left(\mathrm{c}_{2 p}-\mathrm{c}_{2 p}\right)(r)>J_{\text {dec }}$ with equality at $R_{\max }=R_{c c}$ and the entire gate should also be isolated from other donors by this distance. The interaction of ground-state controls in $1 \mathrm{~s}$ should be small: $\mathrm{J}\left(\mathrm{c}_{1 s}-\mathrm{c}_{1 s}\right)(r)<J_{\mathrm{dec}}$ with equality at $R_{\min }^{\prime}$.

\section{B. Constraints on dopant separations from conditions on exchange interaction}

Having defined the conditions that viable entangling gate configurations need to fulfill, we can now find the corresponding distances by calculating maps of the exchange interaction between Si:P and Si:As. The calculation of the exchange-interaction energy using the Heitler-London approximation $[36,50]$, taking into account the multivalley coupled wave function and central cell correction, can be found in Appendix A.

The $2 p \pm$ excited state is split from the $2 p 0$ excited state, owing to the anisotropy of the conduction-band minima, and can therefore be accessed separately using the appropriate wavelength, as is demonstrated experimentally in Ref. [51]. We choose to use the $2 p \pm$ state, as we have calculated that it gives rise to a larger interactive area with the ground-state arsenic than the $2 p 0$ state. We only consider linearly polarized light.

To simplify the treatment of the dopant distributions, we approximate the regions in which $J>J_{\operatorname{dec}}$, for $J$ connecting different types of dopant species in various states shown in Fig. 2, as circles or spheres. To do this, we determine discs whose areas are equal to those of the interaction zones. The radii of these discs are also displayed in the table of Fig. 2. The average fraction of the area that is common to the multivalley delimitation and the circular approximation is 0.88. As we have conserved the total volume, for a two-dopant cluster the method is exact and for a three-dopant cluster this introduces an error which is likely to be less than $12 \%$. Neglecting the multivalley effects would have led to an overestimation of the interactive area.

For two-species gates, we also consider separating the controls and the readouts into two separate parallel planes, sufficiently distant that the ground states do not interact on the timescale of the gate operation but sufficiently close that the qubits can interact with the excited state of the controls. For this to be optimized in a randomly doped sample, the readout layer must be at a distance from the control layer which maximizes exposure to the area in which $J\left(\mathrm{c}_{2 p}-\mathrm{r}_{1 s}\right)(r)<J_{\mathrm{dec}}$ and minimizes exposure to the area in which $J\left(\mathrm{c}_{1 s}-\mathrm{r}_{1 s}\right)(r)>$ $J_{\mathrm{dec}}$, represented in dark blue on Fig. 2. Small periodic oscillations apart, the ground-state interaction exchange energies are equal to $J_{\mathrm{dec}}$ in a spherically symmetric way, and can be modeled as a sphere. It is then clear from basic geometrical considerations, illustrated in Fig. 3, that the optimal distance $d$ separating the two layers should be equal to $R_{\min }$ such that the

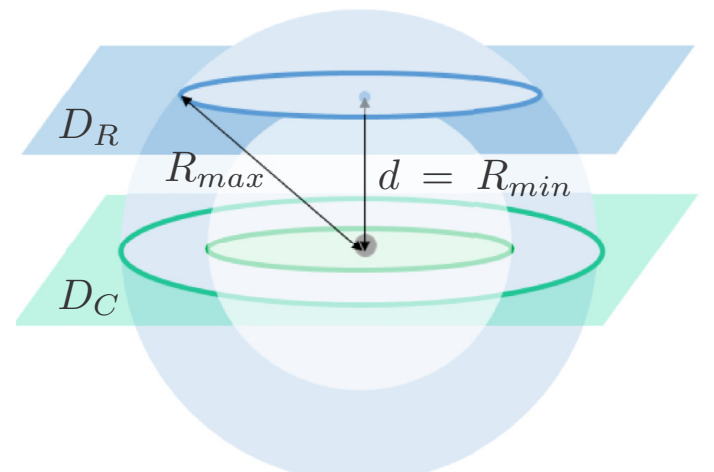

FIG. 3. Parallel bilayers. Separating the 2D distribution of control dopants $D_{c}$ and readout dopants $D_{r}$ into parallel bilayers enables the determination of a distance at which the sphere defined by the conditions on the interaction in Fig. 2 has the most area exposed to the readout distribution. The optimal separation between the layers is $d=R_{\min }$, as can be worked out from basic geometrical considerations.

areas which the qubit can occupy in its own layer correspond to a disk of inner radius 0 and outer radius $\sqrt{R_{\max }^{2}-d^{2}}$, as can be seen in Fig. 3. To ensure that the inner disk has a radius 0 , any multivalley exchange interaction between the $1 s$ state of the Si:As in the upper plane with the $2 p \pm$ states of $\mathrm{Si}: \mathrm{P}$ in the lower plane must be avoided, so we use $d=13.2 \mathrm{~nm}$ (the exact limit on the interaction shown in Fig. 2) in the rest of this paper. To increase the density of entangling gates, the light could be polarized out of plane, thereby reducing the planar interference distance of controls, thus allowing a higher optimum doping density to be used with the result that controls are packed more closely and more qubits become active. All the multivalley exchange interaction limits displayed for the bilayer case in Fig. 2 have been calculated in the readout plane, $13.2 \mathrm{~nm}$ from the control plane and with out-of-plane linearly polarized light.

\section{MAXIMIZING THE ENTANGLING GATE DENSITY}

We aim to tune the free parameters of a randomly doped sample, the doping densities of both species, to maximize the entangling gate density. Phosphorus atoms have a random spatial arrangement on the silicon surface resulting from exposure to phosphine gas [52]. The atoms cannot land in exactly the same location, thus their arrangement corresponds to a hard sphere Poisson point process; however, this radius of exclusion being far smaller than $R_{r r}$, we revert to the use of a pure Poisson point process. We have checked in our Monte Carlo simulation that introducing a small hard sphere radius of up to $0.5 \mathrm{~nm}$, corresponding to the approximate silicon lattice spacing, does not significantly change the results. Many methods, including analytical and simulation-based approaches, are available to treat Poisson point processes: Monte Carlo simulations can treat problems with complex geometries and have the advantage of being flexible, but must be coded efficiently to scale well. Analytical approaches can supply results in closed form but rely on particular assumptions and are valid only if these apply. Analytical nearest-neighbor methods have 
been used to study distributions of $m$ th nearest neighbors [53], isolated pairs of points [54], and the probability of occurrence of reflexive nearest neighbors $[55,56]$.

Here we employ a Monte Carlo simulation to count viable configurations (defined in the previous section). It scales linearly with the number of dopants, i.e., as $\mathcal{O}\left(n_{c}+n_{r}\right)$, where $n_{c}$ is the number of control dopants and $n_{r}$ is the number of readout dopants. This allows large samples (up to a million dopants per run) to be used routinely, leading to lower statistical errors. The algorithm is described in Appendix C. We find agreement of the results with an analytical solution for a Poisson point process in both two and three spatial dimensions. In the Monte Carlo simulation, we are also able to identify configurations with more than two readouts within the viability shell surrounding the same control. These could be useful for implementing multiqubit entangling gates such as the Toffoli gate [57].

\section{A. Independent distributions}

At the point of sample fabrication, each species is separately released onto the silicon surface in gaseous form. The resultant distributions of dopants can be considered to be independent sets of events occupying the same volume. However, not all dopants will form gates, only those complying to the conditions in the table of Fig. 2. The minimum sphere surrounding an isolated control of radius $R_{c c}$ must be empty of other controls. Although this set of isolated events is a subset selected from the full random distribution, we make the approximation that with regard to the readout distribution, the viable controls are randomly distributed.

This sphere of exclusion around viable controls encapsulates and gives clearance to all the distance conditions on the readout distribution, such that the latter acts as an independent distribution within that volume. For example, $R_{c c}$ is over twice the maximum control-readout distance $R_{\max }$, which has the implication that any readout dopant situated between $R_{\min }$ and $R_{\max }$ of a viable control cannot be within $R_{\max }$ of another control and thus, within $T_{1}$, can only interact with one control.

The problem can thereby be distilled into two separate parts: Calculating the density of controls isolated by $R_{c c}$ in the control distribution, and multiplying by the cumulative probability distribution function corresponding to the readout distribution complying to all the distance conditions it is subject to (see Fig. 2).

\section{B. Maximizing the density of events isolated by a fixed radius}

To calculate the optimal total control density, we seek to maximize the density of events isolated by at least a fixed radius $R_{c c}$, which we refer to as the isolated density with isolation radius $R_{c c}$. The probability of finding an event in a spherical shell of thickness $d r$ at a distance $r$ from a randomly chosen event in the distribution of density $D_{t}$ is $\frac{\mathrm{d} V(r)}{\mathrm{d} r} D_{t} d r$, where $V(r)$ is the volume of the sphere of radius $r[58,59]$. The probability that there are no events closer to the randomly chosen event than $r$ is then $P_{0}(r)=e^{-V(r) D_{t}}$, from Refs. [58,60]. Optimizing this probability leads to setting the total density of events to 1 (such that there is no chance of finding any other event within $r$ ), as can be seen in Fig. 4. However, optimizing the density of points $D_{i}$ isolated by exactly $R$ from

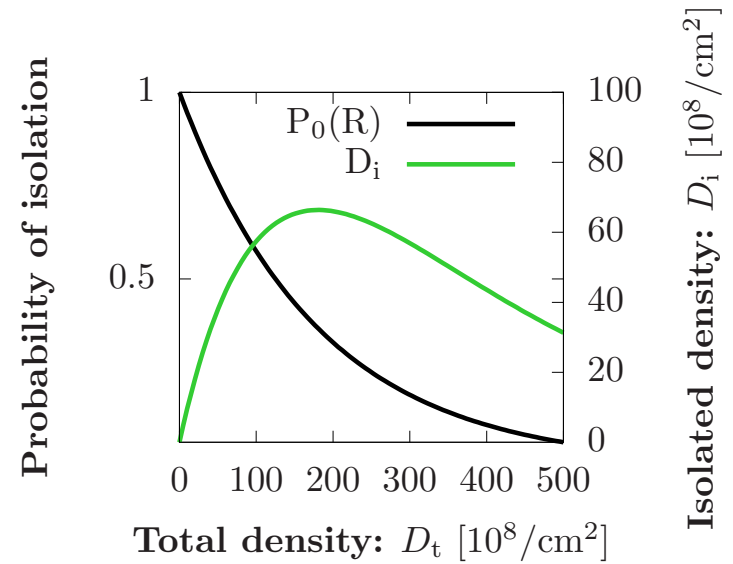

FIG. 4. Comparison of relevant quantites. $\mathrm{P}_{0}(R)$, probability of a random point being isolated by a fixed radius $(42.2 \mathrm{~nm})$, and $D_{\mathrm{i}}$, the density of points isolated by the same fixed radius, as functions of the total density, $D_{\mathrm{t}}$. Whereas the probability doesn't contain a clear maximum to optimize for, the density does.

their neighbors does present a maximum (see Fig. 4).

$$
D_{i} \equiv D_{t} P_{0}(R)=D_{t} e^{-V(R) D_{t}},
$$

which is maximum when

$$
D_{t}\left(D_{i \max }\right)=\frac{1}{V(R)} .
$$

At this density, the fraction of points isolated by $R$ is $1 / e$ :

$$
D_{i, \max }=D_{t} e^{-1} .
$$

The values for the total and isolated control densities corresponding to the control radii (table in Fig. 2) are displayed in Table I.

The main steps of the calculation of the cumulative probability density function using nearest-neighbor methods for the readout distribution can be found in Appendix B. We can now construct the total densities of viable configurations for our three types of entangling gate, for homogeneous doping with a control density $D_{c}$, and a readout density $D_{r}$.

(i) The density of SFG gate configurations is the product of the viable control density with the probability for a control to be surrounded by a successful configuration of readouts [the

TABLE I. Total doping densities giving maximum number of dopants isolated by a radius $R$. Results are for densities in 3D $\left(10^{15} / \mathrm{cm}^{3}\right)$ and in $2 \mathrm{D}\left(10^{10} / \mathrm{cm}^{2}\right)$ for polarizations in plane $e_{\|}$, corresponding to $R=42.2 \mathrm{~nm}$ or polarization out of plane $e_{\perp}$ corresponding to $R=28.5 \mathrm{~nm}$.

\begin{tabular}{lccc}
\hline \hline & $3 \mathrm{D}$ & \multicolumn{2}{c}{$2 \mathrm{D}$} \\
\cline { 3 - 4 } Polarization & Both & $e_{\|}$ & $e_{\perp}$ \\
\hline Total density & 3.18 & 1.79 & 3.91 \\
Isolated density & 1.17 & 0.66 & 1.44 \\
Isolated fraction & $1 / \mathrm{e}$ & $1 / \mathrm{e}$ & $1 / \mathrm{e}$ \\
\hline \hline
\end{tabular}




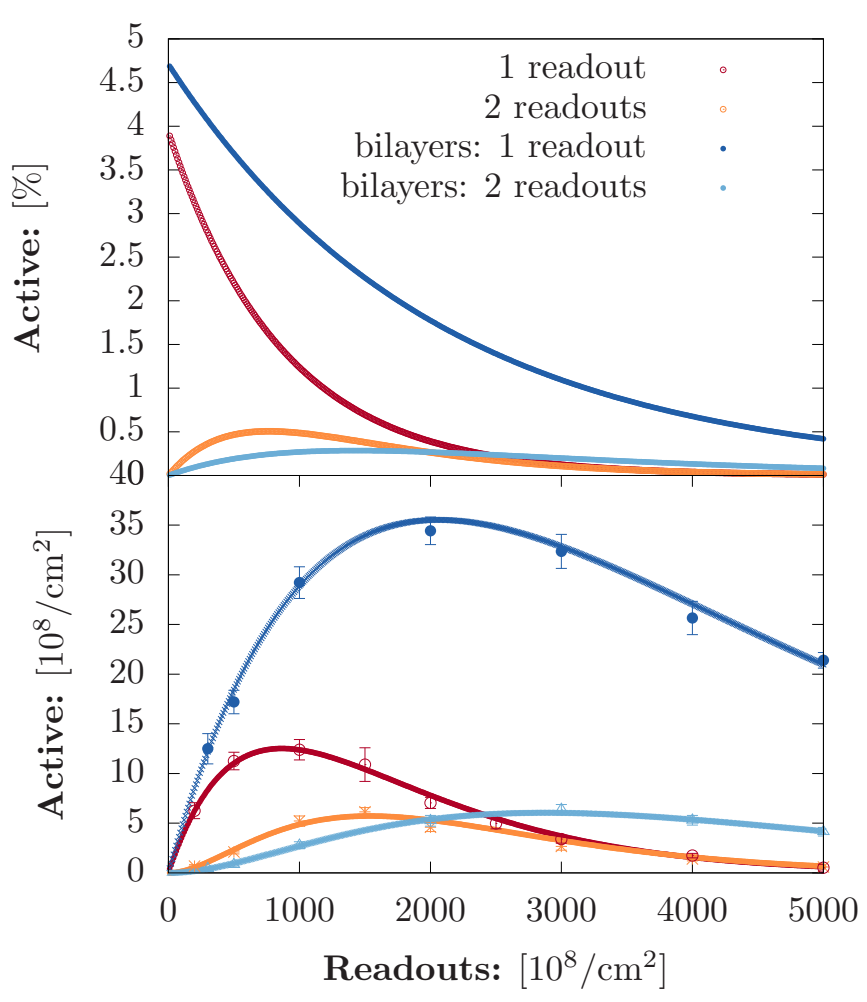

FIG. 5. Dual species doping in 2D-monolayer (reds, polarization in plane)/bilayer (blues, polarization out of plane). Active percentage and readout density as a function of the total readout density in 2D doped silicon. Lines: Poisson point process theory. Error bars: Monte Carlo simulation standard deviation. Orange and light blue: SFG gates (two readouts). Red and dark blue: Excitedground Heisenberg gates (one readout).

product of the CPDFs defined in Eqs. (B1) and (B2)]:

$$
\begin{aligned}
D_{\mathrm{sfg}}\left(D_{c}, D_{r}\right)= & D_{c} e^{-V\left(R_{c c}\right) D_{c}} \int_{R_{\min }}^{R_{\max }} d r_{1} \int_{r_{1}}^{R_{\max }} d r_{2} S\left(r_{1}\right) \tilde{S}\left(r_{2}\right) \\
& \times D_{r}^{2} e^{-\left(V\left(R_{\max }\right)+\delta_{S F G}\right) D_{r}} .
\end{aligned}
$$

(ii) The density of two-species excited-ground Heisenberg entangling gates is

$$
\begin{aligned}
D_{\text {Heis. ex }-\mathrm{gd}}\left(D_{c}, D_{r}\right)= & D_{c} e^{-V\left(R_{c c}\right) D_{c}} \\
& \times \int_{R_{\min }}^{R_{\max }} d r S(r) D_{r}^{2} e^{-\left(V\left(R_{\max }\right)+\delta\right) D_{r}} .
\end{aligned}
$$

(iii) The density of single-species excited-excited Heisenberg entangling gates is

$$
D_{\text {Heis. ex }-\mathrm{ex}}(D)=D \int_{R_{\min }^{\prime}}^{R_{c c}} d r S(r) D^{2} e^{-\left(V\left(R_{c c}\right)+\delta\right) D} .
$$

\section{Results}

The results of Eqs. (7) - (9) for the distances corresponding to Si:P and Si:As in the table of Fig. 2 are plotted in Figs. 5-7. The plotted quantity is the density of readouts which are actively participating in viable entangling gate configurations, i.e., twice the density of entangling gates in the SFG case and

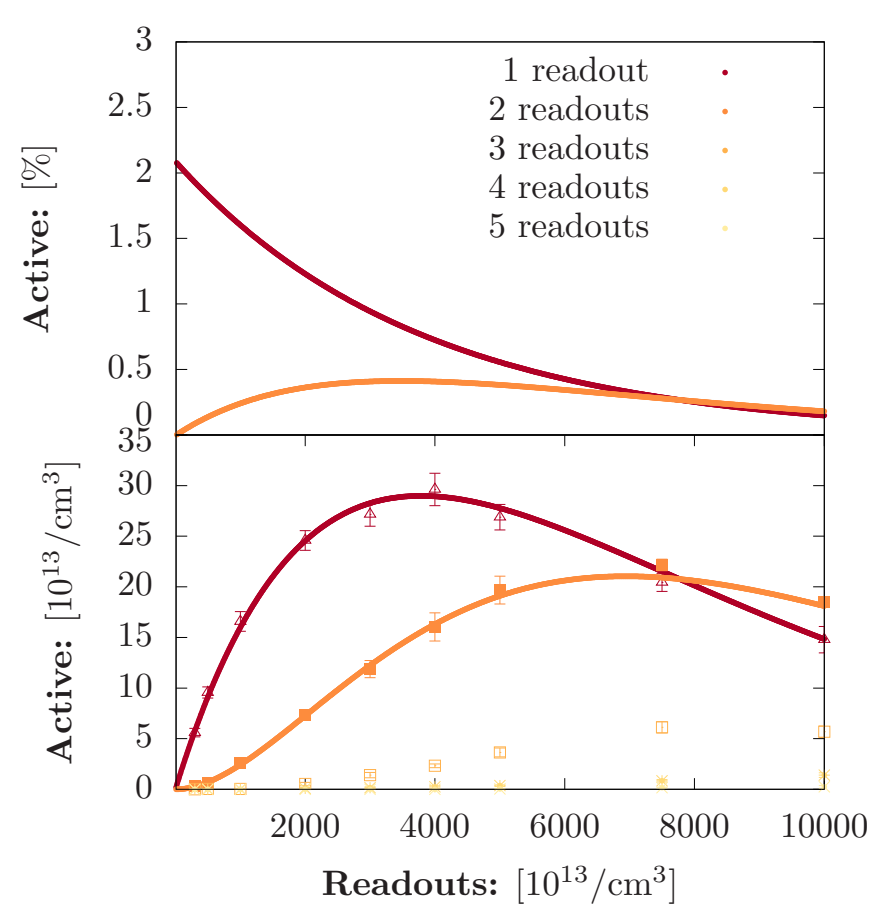

FIG. 6. Dual species doping in 3D. Active percentage and readout density as a function of the total readout density in 3D doped silicon. Lines: Poisson point process theory, Eqs. (7)-(9). Error bars: Monte Carlo simulation standard deviation. Red: Heisenberg gates (one readout). Orange: SFG gates (two readouts). Yellows: Three or more readouts-percentages not shown for these cases because they are negligible.

the same density as that of entangling gates for the Heisenberg single-readout gate cases. The percentage of active readouts is simply the density of readouts which are a part of an entangling gate divided by the total density of readouts.

In all cases, the total density of controls is maximized by choosing $D_{c}=\frac{1}{V\left(R_{c c}\right)}$ from Eq. (5), yielding the viable control density $\frac{e^{-1}}{V\left(R_{c c}\right)}$ from Eq. (6), as can be seen in Fig. 4. The optimal control densities calculated with the distances of the table in Fig. 2 can be seen in Table II .

In $2 \mathrm{D}$, the maximum density of readout dopants (Si:As) which are a part of viable SFG gates is $6 \times 10^{8}$ dopants per $\mathrm{cm}^{2}$, which corresponds to a total readout doping density of $1.5 \times 10^{11}$ dopants per $\mathrm{cm}^{2}$. The bilayer case shows a negligible increase in the density of readouts which are part of SFG gates, for double the total density $\left(3 \times 10^{11}\right.$ dopants per $\mathrm{cm}^{2}$ ). The percentages of readouts involved in viable SFG configurations in both the monolayer and bilayer cases remain below $0.5 \%$.

The maximum density of readout dopants (Si:As) which are a part of viable excited-readout Heisenberg gates is $1.2 \times$ $10^{9}$ dopants per $\mathrm{cm}^{2}$, corresponding to a total density of $8 \times$ $10^{10}$ dopants per $\mathrm{cm}^{2}$ and $1.5 \%$ active readouts. A very clear increase can be seen in the bilayer case. The maximum density of readout dopants, part of viable excited-readout Heisenberg gates in bilayers separated by $13.2 \mathrm{~nm}$, is $3.5 \times 10^{9}$ dopants per $\mathrm{cm}^{2}$, corresponding to $2 \times 10^{11}$ dopants per $\mathrm{cm}^{2}$ and $1.8 \%$ active readouts. 


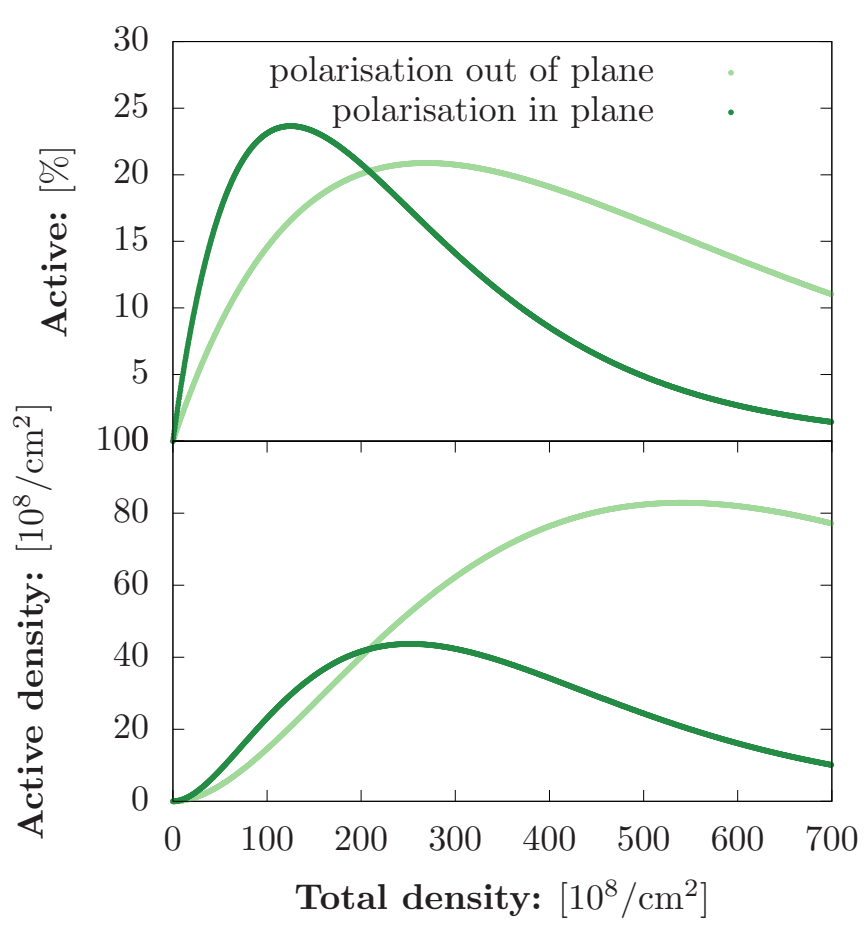

FIG. 7. Single-species Heisenberg gate for both polarizations in a monolayer. The percentage of dopants which are a part of a gate is far larger than in both other gate types discussed. Lines: Poisson point process theory. Error bars: Monte Carlo simulation standard deviation.

The control (Si:P) doping density that achieves maximum density of active readouts is $1.79 \times 10^{10}$ dopants per $\mathrm{cm}^{2}$ in the monolayer case and $3.91 \times 10^{10}$ dopants per $\mathrm{cm}^{2}$ in the bilayer case. It is possible to achieve up to $3.9 \%$ active readouts in the monolayer case and $4.7 \%$ active readouts in the bilayer case for low doping densities. Thus, using equal total densities of Si:P and Si:As leads to some of the highest active percentages of $\mathrm{Si}$ As contributing to excited-ground Heisenberg gates.

The 3D bulk doped results are shown in Fig. 6. The maximum density of readout dopants (Si:As) active in excited-

TABLE II. Analytical (matching simulation) results for total densities of control (P) and readout (As or P) dopants which are part of gates. Densities are in dopants per $\mathrm{cm}^{2}$ (in 2D) or $\mathrm{cm}^{3}$ (in 3D). *Bilayer case: $\mathrm{Si}$ :As in a parallel plane $13.2 \mathrm{~nm}$ from Si:P plane. Polarisation (linear): $e_{\|}$in plane, $e_{\perp}$ out of plane.

\begin{tabular}{lcccccc}
\hline \hline & & Species & $D_{\text {qubits }}$ & $\%$ & $D_{\text {As }}$ & $D_{\mathrm{P}}$ \\
\hline $2 \mathrm{D}$ & $e_{\|}$ & P-As-As & $6 \times 10^{8}$ & $<0.5$ & $1.5 \times 10^{11}$ & $1.8 \times 10^{10}$ \\
& $e_{\|}$ & P-As & $1.2 \times 10^{9}$ & 1.5 & $8 \times 10^{10}$ & $1.8 \times 10^{10}$ \\
& $e_{\|}$ & P-P & $4.3 \times 10^{9}$ & 24 & 0 & $2.5 \times 10^{10}$ \\
& $e_{\perp}$ & P-P & $8.3 \times 10^{9}$ & 21 & 0 & $5.4 \times 10^{10}$ \\
$*$ & $e_{\perp}$ & P-As-As & $6 \times 10^{8}$ & $<0.5$ & $3 \times 10^{11}$ & $3.9 \times 10^{10}$ \\
$*$ & $e_{\perp}$ & P-As & $3.5 \times 10^{9}$ & 1.8 & $2 \times 10^{11}$ & $3.9 \times 10^{10}$ \\
$3 \mathrm{D}$ & & P-As-As & $2 \times 10^{14}$ & $<0.5$ & $7 \times 10^{16}$ & $3.2 \times 10^{15}$ \\
& & P-As & $3 \times 10^{14}$ & 0.8 & $3.5 \times 10^{16}$ & $3.2 \times 10^{15}$ \\
\hline \hline
\end{tabular}

ground Heisenberg type gates is $3 \times 10^{14}$ dopants per $\mathrm{cm}^{3}$, corresponding to a total doping density of readouts of $3.5 \times$ $10^{16}$ dopants per $\mathrm{cm}^{3}$ and $0.8 \%$ active readouts. Similar to the $2 \mathrm{D}$ case, the maximum active readout percentages of $2.1 \%$ can be reached for total readout doping densities comparable to that of the controls $\left(3.2 \times 10^{15}\right.$ dopants per $\left.\mathrm{cm}^{3}\right)$. The maximum density of readout dopants (Si:As) active in SFG type gates is $2 \times 10^{14}$ dopants per $\mathrm{cm}^{3}$ corresponding to a total doping density of readouts of $7 \times 10^{16}$ dopants per $\mathrm{cm}^{3}$. It was possible to gain insights about the densities of gates containing three or more readouts from the Monte Carlo simulation, which may be of interest for different types of quantum gate, e.g., Toffoli gates. Unsurprisingly, they peak at far higher total readout doping densities, such as $7.5 \times$ $10^{16}$ dopants per $\mathrm{cm}^{3}$ for the three readout case (see Fig. 6), but provide lower active readout densities, such as $7 \times 10^{13}$ dopants per $\mathrm{cm}^{3}$ for the same case.

Finally, the single species excited-excited Heisenberg gate in a 2D monolayer yields the highest densities and percentages of active dopants. With in-plane light polarization, 24\% of dopants can be involved in viable excited-excited Heisenberg gates! Active dopant densities of $4.3 \times 10^{9}$ dopants per $\mathrm{cm}^{2}$ can be reached for total doping densities of $2.5 \times 10^{10}$ dopants per $\mathrm{cm}^{2}$. Higher active dopant densities can be reached for out of plane polarization: $8.3 \times 10^{9}$ dopants per $\mathrm{cm}^{2}$ for total doping densities of $5.4 \times 10^{10}$ dopants per $\mathrm{cm}^{2}$. Maximum percentages of $21 \%$ can be achieved with out-of-plane polarization.

\section{PROOF OF PRINCIPLE EXPERIMENT: CONTROL OF FAR-FROM-EQUILIBRIUM MAGNETIZATION DYNAMICS BY ORBITAL EXCITATION}

As a first step toward the implementation of the optically excited exchange entangling gates, we propose a proof of principle experiment to show the control of the magnetization dynamics of the readout ( $\mathrm{Si}: \mathrm{As})$ donor electrons by the control (Si:P) electrons' orbital state, due to enhancement of exchange interactions from orbital excitations. This is the most important building block of the SFG entangling gate. We calculate the quantum many-body magnetization dynamics in the thermodynamic limit using MACE [45], a diagonalization method shown to be well suited for describing the dynamics of randomly placed spins. Because deep donors in silicon act according to the Heisenberg Hamiltonian, this experiment can also be interpreted as a quantum simulation of the twospecies $S=1 / 2$ Heisenberg antiferromagnet ${ }^{1}$ with quenched disorder in the exchange couplings. Note, however, that due to $J_{i j}>0$ for all $i, j$, this model does not show spin-glass phenomenology.

\section{A. Sample fabrication and detection techniques}

Starting from a spin-polarized state, we show below that when all donors are in the orbital ground state, the

\footnotetext{
${ }^{1}$ This has been shown to be an effective low energy description of the half-filled Hubbard model, in the case where the interaction energy between the spins of the lattice is far greater than the hopping strength [61-63].
} 
magnetization of $\mathrm{Si}$ As stays constant on observable timescales, while it changes to a vastly different value when the control species is excited to the $2 p$ state. This implies that while exchange interactions are negligible in the orbital ground state, they are large enough to mediate nontrivial many-body dynamics when in the excited state. Realizing this in experiment would at least partially prove the necessary control over two-body interactions needed for the quantum logic gates discussed in the previous sections.

In experiment, readout of the magnetization of the $\mathrm{Si}: \mathrm{As}$ is done via donor-bound exciton spectroscopy $\left(\mathrm{D}_{0} \mathrm{X}\right.$ spectroscopy) [15,34]. This requires electrical detection, which can be achieved in a dilute 2D layer of $\mathrm{P}$ and As donors using STM hydrogen lithography to pattern highly conductive metallic-doped phosphorus pads into the same plane and overgrowing a protective thin film of crystalline silicon with molecular beam epitaxy. The impurity sheet's metallically doped pads are electrically contacted using electron beam lithography coupled to reactive ion etching to create features which are filled with aluminium using a metal evaporator. The metallic pads are in turn electrically contacted to obtain, in conjunction with terahertz radiation from a FEL $(\lambda=$ $31.6 \mu \mathrm{m}$ for the Si:P $1 s$ to $2 p \pm$ transition) and $\mathrm{D}_{0} \mathrm{X}$ spectroscopy, an electrical signal from the 2D Si:As sheet which is a response to the coherent and nonlinear excitations of the $\mathrm{Si}: \mathrm{P}$ electrons. The sample fabrication and electrical detection technique briefly described above and which we have in mind for the experiment we propose here are described in Ref. [64]. This detection technique enables the precision condensedmatter samples to remain intact after exposure to a FEL pulse.

The contacted impurity layer in silicon is mounted on the bore of a water-cooled Bitter magnet to Zeeman split the ground state impurity electron spin energies, leading to six pairs of dipole-allowed transitions $(\Delta \mathrm{m}=0, \pm 1)$. Electrical detection of electron spin resonance using $\mathrm{D}_{0} \mathrm{X}$ spectroscopy has been demonstrated for magnetic fields of around 0.35 Tesla [33]. A donor-bound exciton can be formed by a direct $1.15 \mathrm{eV}$ photon (the silicon indirect bandgap is of $1.17 \mathrm{eV}$ ). The photon excites an electron from the valence band, leaving behind a hole. When the electron-hole pair recombine via an Auger recombination process, their energy ejects another electron from the donor site, leaving behind a positively charged donor ion [33]. To relax all the electron spin states of both donor species to the lower energy spin state, the sample needs to be cooled down in a dilution fridge to milli-Kelvin temperatures. $\mathrm{D}_{0} \mathrm{X}$ optical pumping then initializes all the readouts to the opposite spin state from the controls (and later to read out the occupation of one of the Si:As spin states).

\section{B. Experimental proposal}

Following the techniques described previously, each electron has the initial spin state $\left|S_{i}\right\rangle$ with

$$
\left|S_{i}\right\rangle= \begin{cases}|\uparrow\rangle & \text { if } \quad i=\text { readout } \\ |\downarrow\rangle & \text { if } \quad i=\text { control, }\end{cases}
$$

and the whole system is initially in a product state $\left|\psi_{0}\right\rangle$ such that

$$
\left|\psi_{0}\right\rangle=\left|S_{1} S_{2} \ldots S_{n}\right\rangle
$$

When the terahertz frequency FEL is switched on, it illuminates the entire sample and excites the control ( $\mathrm{Si}: \mathrm{P})$ electrons to the $2 p \pm$ state. As mentioned previously, the experimentally demonstrated decay time of 3D bulk doped Si:P in the $2 p \pm$ state in nonisotopic silicon is 200 ps [47], which gives the timescale available to our experiment.

The dopants evolve in time according to the Heisenberg Hamiltonian,

$$
\begin{aligned}
\mathcal{H}= & \frac{1}{2} \sum_{i \neq j} J_{i j}^{\mathrm{AsAs}} \mathbf{S}_{i}^{\mathrm{As}} \mathbf{S}_{j}^{\mathrm{As}}+\frac{1}{2} \sum_{i \neq j} J_{i j}^{P A s} \mathbf{S}_{i}^{P} \mathbf{S}_{j}^{\mathrm{As}} \\
& +\frac{1}{2} \sum_{i \neq j} J_{i j}^{P P} \mathbf{S}_{i}^{P} \mathbf{S}_{j}^{P},
\end{aligned}
$$

where we made the species dependence of the interactions explicit. The strength of the interaction $J^{P A s}, J^{P P}$ depends explicitly on the orbital state of the control species (Si:P) (see Appendix A). If the densities are high enough, the Si:P wave functions will overlap with the Si:As and lead to nontrivial dynamics.

The average magnetization of the readouts ( $\mathrm{Si}: \mathrm{As})$ is given by

$$
\left\langle S^{z \mathrm{As}}(t)\right\rangle=\left\langle\psi_{0}\left|\frac{1}{N} \sum_{i} S_{i}^{z \mathrm{As}}(t)\right| \psi_{0}\right\rangle .
$$

The average spin flip probability is then defined as

$$
\begin{aligned}
P_{\mathrm{sf}}^{\mathrm{As}}(t) & =\frac{1}{N} \sum_{i}\langle\mid \uparrow\rangle\left\langle\left.\uparrow\right|_{i}\right\rangle \\
& =\frac{1}{2 N}+\left\langle S^{\mathrm{As}}(t)\right\rangle,
\end{aligned}
$$

where $|\uparrow\rangle\left\langle\left.\uparrow\right|_{i}\right.$ is the projector onto the $i$ th spin being in spin up.

To make a differential measurement, we compare the average spin-flip probability of the readout dopants when the control dopants are in the ground state with the average spin flip probability when the control dopants are collectively excited to the $2 p \pm$ state. As a result, we then expect

$$
P_{\mathrm{sf}}^{\mathrm{As}}(t) \rightarrow\left\{\begin{array}{ll}
\approx 0 & \text { for control in } 1 s \\
\neq 0 & \text { for control in } 2 p .
\end{array}, \text { for late times } \mathrm{t}\right.
$$

We can deduce that the nontrivial dynamics of the spinflip probability must have been due to interactions between species as the species themselves are in an eigenstate of their respective Heisenberg Hamiltonian.

In the following, we will now predict the outcome of the experiment using an exact diagonalization technique.

\section{Dynamics of the arsenic spin flip probability within MACE}

To simulate the experimental outcome, i.e., the magnetization dynamics, we use the MACE technique, which has been shown to capture the magnetization dynamics in disordered long-range interacting quantum spin systems realized by cold dipolar molecules [45] and Rydberg atoms [65].

MACE assumes that in a system where spins are randomly placed in space, contributions to the local magnetization dynamics of a particular spin are only made by the spins with which it has the largest interaction-exchange energies. 


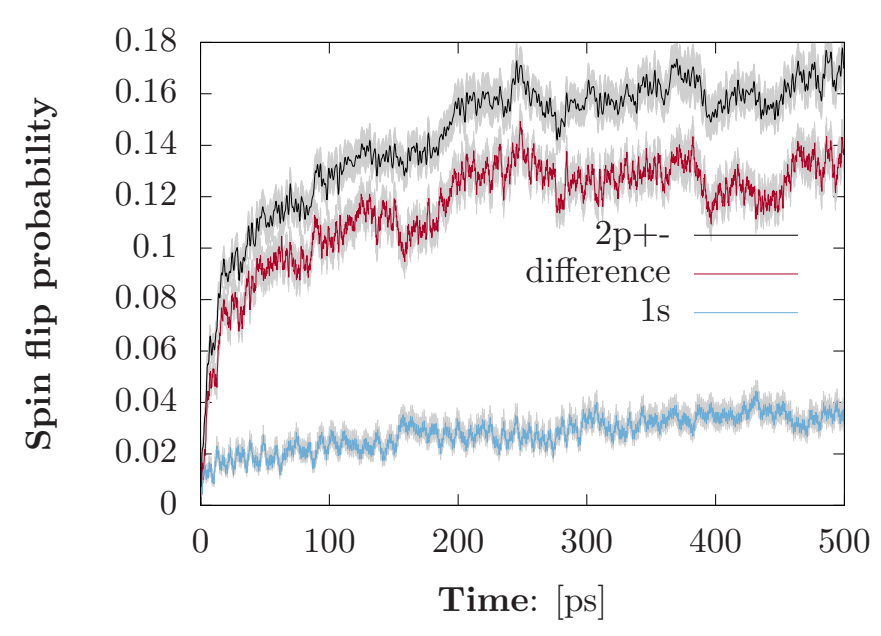

FIG. 8. Average spin-flip probability of the Si:As for Si:P and Si:As densities given by $7 \times 10^{9}$ dopants per $\mathrm{cm}^{2}$ as obtained with MACE, where both species are in the same 2D layer. Si:As is always in the $1 s$ ground state. Light blue: $\mathrm{P}$ in the $1 s$ ground state, dark blue: $\mathrm{P}$ in the $2 p \pm$ excited state, red: The absolute value of the difference between both curves. The results are averaged over 400 clusters of size 8 and the shaded areas around the curves are the standard deviation estimated with a jackknife binning analysis of the cluster sampling.

Therefore, it is enough to exactly diagonalize this cluster and then average over all such clusters. Convergence is then sought by increasing the cluster size. In our system, convergence was found for cluster sizes 5 and above due to the short-range nature of the interactions. This is in contrast to the larger cluster sizes needed in algebraically decaying interactions [66]. Plotted on Fig. 8 are results from cluster size 8 with jackknife error estimates from the quenched disorder sampling in grey [67].

We calculated that there is no visible change in the magnetization of the arsenic for fields up to 10 Tesla. We have also checked that there are no significant differences between defining clusters with the largest exchange interactions or the nearest neighbors of the spin of interest.

The difference in the dynamics of the Si:As spin-flip probability between $\mathrm{Si}: \mathrm{P}$ being in the orbital ground state or the excited state can be seen in Fig. 8 to be $13 \%$ within the 200ps experimentally detected decoherence time of the Si:P $2 p \pm$ excited state, for doping densities of both As and $\mathrm{P}$ of $7 \times 10^{9}$ dopants per $\mathrm{cm}^{2}$, which is accessible with current doping techniques.

This shows that already with only global measurements at hand, nontrivial quantum many-body dynamics could be probed within this dual-doped system. Comparing measurements where $\mathrm{Si}: \mathrm{P}$ is in the ground and excited state would furthermore show that substantial control over the dynamics of the Si:As spins can be implemented within this scheme, therefore showing that one of the prime requirements of the SFG entangling gate are within reach of current experimental platforms.

\section{CONCLUSION}

In this paper, we have established the optimum densities of optically controlled entangling gates achievable in randomly doped silicon. To determine which spacing between dopant electrons can realistically create entangling gates, we created spatial maps of the Heisenberg interaction exchange energy between same-species and dual-species dopants. We focused on $\mathrm{Si}: \mathrm{P}$ and $\mathrm{Si}:$ As because they are, to date, the most wellunderstood dopants for diffusion onto a silicon surface in ultrahigh vacuum.

Respecting these conditions, we obtained matching results in both the nearest-neighbor treatment of a Poisson point process and a Monte Carlo simulation. The maximally achievable densities of entangling gates in 2D are in the low $10^{9}$ dopants per $\mathrm{cm}^{2}$ and in 3D are in the low $10^{14}$ dopants per $\mathrm{cm}^{3}$. This corresponds to relatively low percentages of active dopants. An increase was found in the density of gates when the dopants are divided into two parallel layers. Furthermore, by considering same-species Heisenberg gates, up to $24 \%$ of dopants could be involved in viable gate structures. We also showed that in 3D there arise situations in which gates with three and four qubits reach significant densities.

We proposed a proof of principle experiment aimed at demonstrating the onset of two-body (entangling) interactions caused by the optical excitation of one of the species in a $2 \mathrm{D}$ randomly doped sample. The far-from-equilibrium magnetization dynamics of the $\mathrm{Si}$ :As donor electron spins were calculated using the MACE technique, depending on the orbital state of the Si:P donor electrons. For low but equal densities of Si:P and Si:As, we found a large difference in the time evolution of the spin-flip probability of around $13 \%$ between the cases where phosphorus was in the ground or excited state, showing that such an experiment would indeed be feasable.

This work can be extended in various ways. Single-donor placement techniques such as hydrogen lithography could be used to create two or three dopant structures in silicon to verify the maps of the exchange interaction energy calculated here. The theory we have developed can be applied to different dopant species such as selenium or acceptors such as boron, and could be modified to accommodate hard-sphere configurations and would be relevant for Rydberg atoms which deviate from pure Poissonian statistics in the blockaded regime [68].

\section{ACKNOWLEDGMENTS}

We thankfully acknowledge discussions with R. Crane, N. J. Curson, G. Matmon, B. N. Murdin, and A. Kolker. A.S. thanks the London Centre for Nanotechnology for hospitality. We gratefully acknowledge financial support from the UK Engineering and Physical Sciences Research Council (COMPASSS/ADDRFSS, Grant No. EP/M009564/1). A.S. acknowledges financial support from the International Max Planck Research School for Quantum Science and Technology (IMPRS-QST) funded by the Max Planck Society (MPG).

\section{APPENDIX A: EXCHANGE CALCULATION WITH MULTIVALLEY WAVE FUNCTIONS}

To calculate the exchange interaction between two donors, we use the Heitler-London approximation [36]

$$
\begin{aligned}
J= & \int d \boldsymbol{r}_{1} d \boldsymbol{r}_{2} \psi_{1}^{*}\left(\boldsymbol{r}_{1}\right) \psi_{2}^{*}\left(\boldsymbol{r}_{2}-\boldsymbol{R}\right) \frac{e^{2}}{4 \pi \epsilon_{0} \epsilon_{r}\left|\boldsymbol{r}_{1}-\boldsymbol{r}_{2}\right|} \\
& \times \psi_{2}\left(\boldsymbol{r}_{1}-\boldsymbol{R}\right) \psi_{1}\left(\boldsymbol{r}_{2}\right),
\end{aligned}
$$




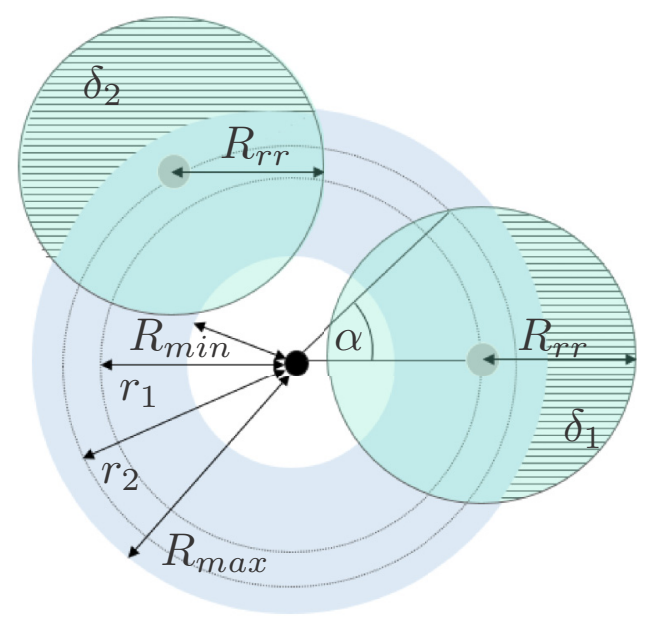

FIG. 9. Schematic representations of geometric considerations for readout dopants surrounding the control dopant. The first- and second-nearest neighbors of a random point chosen in the readout distribution must be separated by $R_{r}$, which is calculated using the exclusion angle $\alpha$ and the hashed areas referred to as $\delta$.

where $\boldsymbol{R}=\boldsymbol{R}_{2}-\boldsymbol{R}_{1}$ is the separation vector between the two donors and $\epsilon_{r}=11.4$ is the dielectric constant of silicon. The wave function of each donor can be either the $1 s \mathrm{~A}_{1}$ ground state, the $2 p_{0}$ or $2 p_{ \pm}$excited states. In the multivalley effective mass theory, all of these wave functions can be expanded as

$$
\psi^{(j)}(\boldsymbol{r})=\sum_{\mu} F_{\mu}^{(j)}(\boldsymbol{r}) \phi_{\mu}(\boldsymbol{r}),
$$

where $j=1,2,3$ indicates the $1 s \mathrm{~A}_{1}$ ground state, the $2 p_{0}$ and $2 p_{ \pm}$excited states, respectively; $\mu= \pm x, \pm y, \pm z$ indicates the valleys of silicon's conduction band minima, $\phi_{\mu}(\boldsymbol{r})=$ $e^{i \boldsymbol{k}_{\mu} \cdot \boldsymbol{r}} u_{\mu}(\boldsymbol{r})$ are the Bloch functions at the minima, $\left|\boldsymbol{k}_{\mu}\right|=$ $0.84 \times 2 \pi / a_{0}$ where $a_{0}=0.543 \mathrm{~nm}$ is the lattice constant. The $F_{\mu}(\boldsymbol{r})$ are the envelope functions. For the $1 s \mathrm{~A}_{1}$ state, the envelope function of the $+z$ valley is [69]

$$
F_{+z}^{(1)}(\boldsymbol{r})=\frac{\exp \left[-\left(\frac{x^{2}+y^{2}}{\left(\alpha a_{1}\right)^{2}}+\frac{z^{2}}{\left(\alpha b_{1}\right)^{2}}\right)^{1 / 2}\right]}{\sqrt{6 \pi\left(\alpha a_{1}\right)^{2}\left(\alpha b_{1}\right)}},
$$

where $a_{1}=2.42 \mathrm{~nm}$ and $b_{1}=1.39 \mathrm{~nm}$. The factor $\alpha$ accounts for the contraction of the ground state [70] due to the central cell correction (CCC) and is given by $\alpha=\sqrt{E_{\mathrm{SV}} / E_{B}}$ where

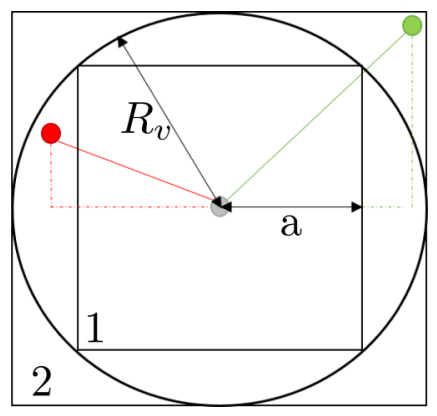

FIG. 10. Monte Carlo simulation isolation checking algorithm described in Algorithm 1.
Algorithm 1 Check isolation of a point by $R_{v}$ (squares are labeled in Fig. 10).

Require: $D$ : Array containing coordinates of all points in distribution

Require: $P$ : Array containing coordinates of point being tested for isolation

$$
a \leftarrow R_{v} \cos 45
$$

$\mathrm{i} \leftarrow 0$

Mark all elements of $D$ as viable

while $i<$ length $(D)$ do

if $P$ is marked as viable then

$$
\begin{aligned}
& \text { if } P_{x}-a<D[i]_{x}<P_{x}+a \text { then } \\
& \text { if } P_{y}-a<D[i]_{y}<P_{y}+a \text { then } \\
& \text { Mark } D[i] \text { and } P \text { as nonviable } \\
& \quad i=i+1 \\
& \text { end if } \\
& \text { end if } \\
& \text { if } P_{x}-R_{v}<D[i]_{x}<P_{x}+R_{v} \text { then } \\
& \text { if } P_{y}-R_{v}<D[i]_{y}<P_{y}+R_{v} \text { then } \\
& \quad b \leftarrow S q r t\left(\left(D[i]_{x}-P_{x}\right)^{2}+\left(D[i]_{y}-P_{y}\right)^{2}\right) \\
& \text { if } b<R_{v} \text { then } \\
& \quad \text { Mark } D[i] \text { and } \mathrm{P} \text { as nonviable } \\
& \quad i=i+1 \\
& \quad \text { end if } \\
& \text { end if } \\
& \text { end if } \\
& i=i+1 \\
& \text { end if } \\
& \text { end while }
\end{aligned}
$$

$E_{\mathrm{SV}}=29.7 \mathrm{meV}$ is the binding energy obtained from a singlevalley theory without the CCC, as in Ref. [69], and $E_{B}$ is the experimental binding energy which is $45.58 \mathrm{meV}$ for $\mathrm{Si}: \mathrm{P}$ and $53.77 \mathrm{meV}$ for Si:As [51]. The other envelope functions are obtained by using $F_{\mu}=F_{-\mu}$ and cyclic permutations of $x, y, z$.

The excited state energies and wave functions of Si:P are identical to those of Si:As [51,71], and are dependent on the polarization of the light field. For polarization with the unit vector $\boldsymbol{\epsilon}=\left[\epsilon_{x}, \epsilon_{y}, \epsilon_{z}\right]$, the excited states are also given by Eq. (A2) but the envelope functions are now

$$
\begin{aligned}
& F_{+z}^{(2)}\left(\boldsymbol{r}=\frac{\epsilon_{z} z}{\sqrt{2 \pi a_{2}^{2} b_{2}^{3}}} \exp \left[-\sqrt{\frac{x^{2}+y^{2}}{a_{2}^{2}}+\frac{z^{2}}{b_{2}^{2}}}\right],\right. \\
& F_{+z}^{(3)}\left(\boldsymbol{r}=\frac{\epsilon_{x} x+\epsilon_{y} y}{\sqrt{4 \pi a_{3}^{4} b_{3}}} \exp \left[-\sqrt{\frac{x^{2}+y^{2}}{a_{3}^{2}}+\frac{z^{2}}{b_{3}^{2}}}\right],\right.
\end{aligned}
$$

where $a_{2}=3.68 \mathrm{~nm}, b_{2}=2.23 \mathrm{~nm}, a_{3}=5.45 \mathrm{~nm}$, and $b_{3}=$ $3.35 \mathrm{~nm}$. The other envelope functions can again be derived using $F_{\mu}=F_{-\mu}$ and cyclic permutations of $x, y, z$.

We can further expand the Bloch function in Eq. (A2) in terms of plane waves as $\phi_{\mu}(\boldsymbol{r})=\sum_{\boldsymbol{G}} c_{\boldsymbol{G}} e^{i \boldsymbol{G} . \boldsymbol{r}}$ where $\boldsymbol{G}$ is the reciprocal lattice vector. Substituting this into Eq. (A2) and then Eq. (A1), neglecting the fast oscillating terms in the resulting integrand, and using the equality $\sum_{\boldsymbol{G}}\left|c_{\boldsymbol{G}}\right|^{2}=1$, we 


\section{Haystack}

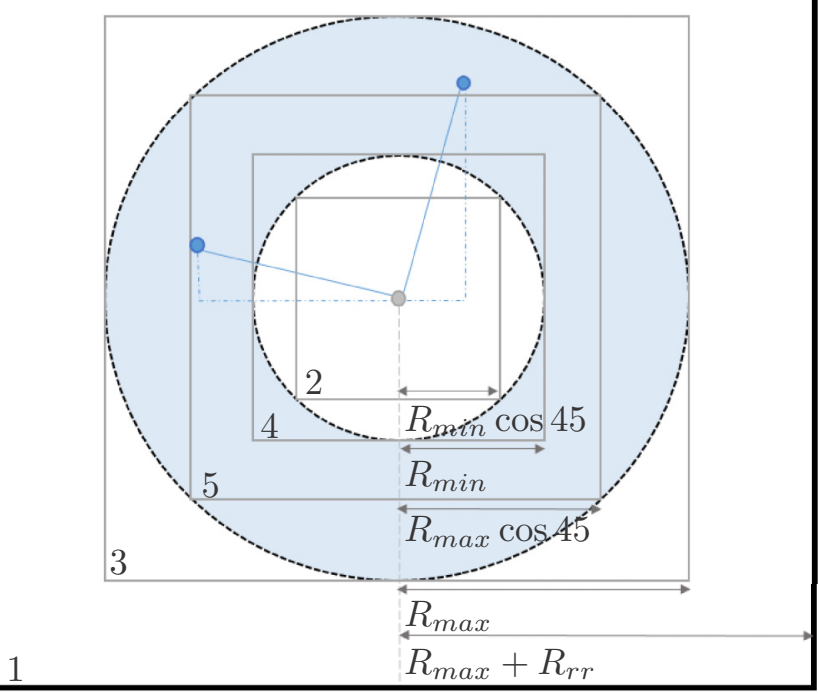

FIG. 11. Schematic explanation of the haystack algorithm described in Algorithm 2. In the case of there being many readouts in one shell, this method of recording reciprocal nonviability cuts calculation time approximately in half.

arrive at

$$
J=2 \sum_{\mu, \nu} j_{\mu, \nu} \cos \left(\boldsymbol{k}_{\mu} \cdot \boldsymbol{R}\right) \cos \left(\boldsymbol{k}_{v} \cdot \boldsymbol{R}\right),
$$

where

$$
\begin{aligned}
j_{\mu, \nu}= & \int d \boldsymbol{r}_{1} d \boldsymbol{r}_{2} F_{1, \mu}^{*}\left(\boldsymbol{r}_{1}\right) F_{2, \nu}^{*}\left(\boldsymbol{r}_{2}-\boldsymbol{R}\right) \frac{e^{2}}{4 \pi \epsilon_{0} \epsilon_{r}\left|r_{1}-r_{2}\right|} \\
& \times F_{2, \nu}\left(\boldsymbol{r}_{1}-\boldsymbol{R}\right) F_{1, \mu}\left(\boldsymbol{r}_{1}\right) .
\end{aligned}
$$

These highly oscillating integrals are evaluated numerically with the Vegas PaCKage [72].

\section{APPENDIX B: READOUT CUMULATIVE PROBABILITY DENSITY FUNCTION CALCULATION}

Let $S(r)=\frac{\mathrm{d} V(r)}{\mathrm{d} r}$ be the surface area of the $n$-dimensional sphere of radius $r$ [i.e., $S(r)=2 \pi r$ in 2D and $S(r)=4 \pi r^{2}$ in $3 \mathrm{D}]$.

If the readout density is $D_{r}$, the probability of finding the first- and second-nearest neighbors between $R_{\min }$ and $R_{\max }$ is

$$
\int_{R_{\min }}^{R_{\max }} d r_{1} \int_{r_{1}}^{R_{\max }} d r_{2} S\left(r_{1}\right) S\left(r_{2}\right) D_{r}^{2} e^{-V\left(R_{\max }\right) D_{r}} .
$$

However, the number of viable configurations is reduced by the additional requirement that the second-nearest neighbor must be at least $R_{r r}$ from the first. If $r_{2}<\left(r_{1}+R_{r r}\right)$, the sphere of radius $r_{2}$ defining the viable positions for the second-nearest neighbor must therefore have a spherical cap removed from it (see Fig. 9) subtending an angle $\alpha=$ $\cos ^{-1}\left(\frac{r_{1}^{2}+r_{2}^{2}-R_{r r}^{2}}{2 r_{1} r_{2}}\right)$. This gives rise to a new surface of smaller
Algorithm 2 Find which controls and readouts are active (squares are labeled in Fig. 11).

Require: $C_{v}$ : Array containing all viable controls (isolated by $R_{c c}$ within the control distribution: the output of Algorithm 1)

Require: Mark all elements of $C$ as active

Require: $R$ : Array containing all readouts

Require: Mark all elements of $R$ as nonactive

$\mathbf{i} \leftarrow 0$

while $i<\operatorname{Length}\left(C_{v}\right)$ do

array $H \leftarrow$ elements of $R$ located within square 1 of $C_{v}[i]$

if $H$ does not contain elements within square 3 then

Mark $C_{v}[i]$ as nonactive

$$
i=i+1
$$

else

if $H$ contains any element within square 2 then

Mark $C_{v}[i]$ as nonactive

$$
i=i+1
$$

else

for $k$ indexing elements of $H$ within square 4 do

if $R[k]$ within $R_{\min }$ (Pythagoras calculation) then Mark $C_{v}[i]$ as nonactive

$i=i+1$

else

Check $R[k]$ for isolation within $H$ (Algorithm 1) and, if isolated, mark $R[k]$ as active. If active, array

$$
R_{a} \leftarrow+R[k]
$$

end if

end for

for $k$ indexing of elements within square 5 and not within square 4 do

Check $R[k]$ for isolation within $H$ (Algorithm 1) \& if isolated, mark $R[k]$ as active. If active, array

$$
R_{a} \leftarrow+R[k]
$$

end for

for $k$ indexing elements of $\mathrm{H}$ between squares 3 and 5 do if $R[k]$ within $R_{\max }$ (Pythagoras calculation) then

Check $R[k]$ for isolation within $H$ (Algorithm 1) and, if isolated, mark $R[k]$ as active. If active, array

$$
R_{a} \leftarrow+R[k]
$$

end if

end for

if $\operatorname{Size}\left(R_{a}\right)>0$

Mark $C_{v}[i]$ as having Length $\left(R_{a}\right)$ readouts

$$
i=i+1
$$

end if

end if

end if

end while

area $\tilde{S}\left(r_{2}\right)=2(\pi-\alpha) r_{2}$ in $2 \mathrm{D}$ and $\tilde{S}\left(r_{2}\right)=4(\pi-\alpha) r_{2}^{2}$ in 3D. In the bilayer case, $R_{\min }=0$ which further constrains $\alpha$ to be $\pi$ if $r_{1}<\left(R_{r r}-R_{\max }\right)$, i.e., if $\alpha$ is complex.

Finally, cases where further readout donors lie outside the sphere of radius $R_{\max }$ but within a volume $\delta$ defined as being within radius $R_{r r}$ of the first- or second-nearest neighbors must be excluded; this corresponds to reducing the probability by a factor

$$
e^{-\delta D_{r}}
$$


In the SFG case, if $\delta_{1}\left(r_{1}\right)$ and $\delta_{2}\left(r_{2}\right)$ are the volumes of the spheres of radius $R_{r r}$ centered on the first- and second-nearest neighbors which lie outside the sphere of volume $R_{\max }$, and $\delta_{o v}\left(r_{1}, r_{2}, \theta\right)$ is the overlap between these two volumes where $\theta$ is the angle between the readouts, $\delta_{\mathrm{SFG}}=\delta_{1}\left(r_{1}\right)+\delta_{2}\left(r_{2}\right)-$ $\delta_{o v}\left(r_{1}, r_{2}, \theta\right)$. In both the other gate types, $\delta=\delta_{1}\left(r_{1}\right)$. This three-circle overlap configuration and area calculation has been made into a Wolfram Demonstrations Project [73] and the functions corresponding to the overlap of two and three circles case were taken from Ref. [74].

\section{APPENDIX C: MONTE CARLO SIMULATION}

The MonteCarlo simulation was written in PHP with a MySQLi database. The front end is written in HTML/CSS so simulations can be run through a browser and made available on the internet. It scales optimally with the number of dopants, i.e., $\mathrm{O}\left(\mathrm{n}_{c}+\mathrm{n}_{\mathrm{r}}\right)$, where $\mathrm{n}_{\mathrm{c}}$ is the number of control dopants and $\mathrm{n}_{\mathrm{r}}$ is the number of readout dopants, such that it allows for computations on large densities and such that the wait time is minimal. Optimal scaling stems principally from the avoidance of full Pythagoras computations. This is achieved with four different techniques. In regard to the first step in the whole algorithm, which is to analyze the controls to identify all viable controls, the avoidance of Pythagoras is achieved by first partitioning the space according to the expected mean nearest-neighbor distance, second by using Pythagoras only for points lying between the enclosing squares (Fig. 10), third by skipping to the next point as soon as conditions are found to be breached, and fourth by reciprocating information between adjacent points.

With regard to the second step, which is to work within the viable control distribution and the full readout distribution, avoidance of Pythagoras is achieved by using the search sequence represented diagrammatically in Fig. 11, referred to as a haystack search.

Only the readouts close enough to a viable control and which might therefore constitute or interfere with an entangling gate are analyzed. Consequently, slightly more than half the readout dopants are never involved in the algorithm.
[1] H. Häffner, C. F. Roos, and R. Blatt, Phys. Rep. 469, 155 (2008).

[2] J. Zhang, G. Pagano, P. W. Hess, A. Kyprianidis, P. Becker, H. Kaplan, A. V. Gorshkov, Z.-X. Gong, and C. Monroe, Nature (London) 551, 601 (2017).

[3] M. Greiner, O. Mandel, T. Esslinger, T. W. Hansch, and I. Bloch, Nature (London) 415, 39 (2002).

[4] C. Gross and I. Bloch, Science 357, 995 (2017).

[5] A. Mazurenko, C. S. Chiu, G. Ji, M. F. Parsons, M. KanászNagy, R. Schmidt, F. Grusdt, E. Demler, D. Greif, and M. Greiner, Nature (London) 545, 462 (2017).

[6] H. Bernien, S. Schwartz, A. Keesling, H. Levine, A. Omran, H. Pichler, S. Choi, A. S. Zibrov, M. Endres, M. Greiner, V. Vuletić, and M. D. Lukin, Nature (London) 551, 579 (2017).

[7] J. Sau, Physics 10, 68 (2017).

[8] V. Lahtinen and J. K. Pachos, Sci. Post. Phys. 3, 021 (2017).

[9] M. H. Devoret, A. Wallraff, and J. M. Martinis, arXiv:condmat/0411174.

[10] R. Barends, J. Kelly, A. Megrant, A. Veitia, D. Sank, E. Jeffrey, T. C. White, J. Mutus, A. G. Fowler, B. Campbell, Y. Chen, Z. Chen, B. Chiaro, A. Dunsworth, C. Neill, P. O'Malley, P. Roushan, A. Vainsencher, J. Wenner, A. N. Korotkov, A. N. Cleland, and J. M. Martinis, Nature (London) 508, 500 (2014).

[11] J. Kelly, R. Barends, A. G. Fowler, A. Megrant, E. Jeffrey, T. C. White, D. Sank, J. Y. Mutus, B. Campbell, Y. Chen, Z. Chen, B. Chiaro, A. Dunsworth, I.-C. Hoi, C. Neill, P. J. J. O’Malley, C. Quintana, P. Roushan, A. Vainsencher, J. Wenner, A. N. Cleland, and J. M. Martinis, Nature 519, 66 (2015).

[12] L. Childress and R. Hanson, MRS Bull. 38, 134 (2013).

[13] X. Mi, J. V. Cady, D. M. Zajac, J. Stehlik, L. F. Edge, and J. R. Petta, Appl. Phys. Lett. 110, 043502 (2017).

[14] C. D. Hill, E. Peretz, S. J. Hile, M. G. House, M. Fuechsle, S. Rogge, M. Y. Simmons, and L. C. L. Hollenberg, Sci. Adv. 1, e1500707 (2015).

[15] G. W. Morley, Electron Paramagnetic Resonance: Volume 24 (The Royal Society of Chemistry, 2015), pp. 62-76.
[16] P. W. Shor, Proceedings of the 35th Annual Symposium on Foundations of Computer Science SFCS '94 (IEEE, Piscataway, NJ, 1994), p. 124.

[17] L. K. Grover, Proceedings of the 28th Annual ACM Symposium on Theory of Computing STOC '96 (ACM, New York, 1996), p. 212.

[18] J. Biamonte, P. Wittek, N. Pancotti, P. Rebentrost, N. Wiebe, and S. Lloyd, Nature (London) 549, 195 (2017).

[19] R. P. Feynman, Int. J. Theor. Phys. 21, 467 (1982).

[20] A. D. King, J. Carrasquilla, J. Raymond, I. Ozfidan, E. Andriyash, A. Berkley, M. Reis, T. Lanting, R. Harris, F. Altomare, K. Boothby, P. I. Bunyk, C. Enderud, A. Fréchette, E. Hoskinson, N. Ladizinsky, T. Oh, G. Poulin-Lamarre, C. Rich, Y. Sato, A. Y. Smirnov, L. J. Swenson, M. H. Volkmann, J. Whittaker, J. Yao, E. Ladizinsky, M. W. Johnson, J. Hilton, and M. H. Amin, Nature (London) 560, 456 (2018).

[21] J. Braumüller, M. Marthaler, A. Schneider, A. Stehli, H. Rotzinger, M. Weides, and A. V. Ustinov, Nat. Commun. 8, 779 (2017).

[22] L. Lamata, A. Parra-Rodriguez, M. Sanz, and E. Solano, Adv. Phys.: X 3, 1457981 (2018)

[23] D. DiVincenzo and D. Loss, Superlattices Microstruct. 23, 419 (1998).

[24] B. E. Kane, Nature (London) 393, 133 (1998).

[25] J. J. L. Morton, A. M. Tyryshkin, R. M. Brown, S. Shankar, B. W. Lovett, A. Ardavan, T. Schenkel, E. E. Haller, J. W. Ager, and S. A. Lyon, Nature (London) 455, 1085 (2008).

[26] A. M. Stoneham, A. J. Fisher, and P. T. Greenland, J. Phys. Condens. Matter 15, L447 (2003).

[27] R. Rodriquez, A. J. Fisher, P. T. Greenland, and A. M. Stoneham, J. Phys.: Condens. Matter 16, 2757 (2004).

[28] J. J. Pla, K. Y. Tan, J. P. Dehollain, W. H. Lim, J. J. L. Morton, F. A. Zwanenburg, D. N. Jamieson, A. S. Dzurak, and A. Morello, Nature (London) 496, 334 (2013).

[29] K. Saeedi, S. Simmons, J. Z. Salvail, P. Dluhy, H. Riemann, N. V. Abrosimov, P. Becker, H.-J. Pohl, J. J. L. Morton, and M. L. W. Thewalt, Science 342, 830 (2013). 
[30] I. K. Saeedi, Optical NMR study of 31P donor spins in isotopically enriched 28Si, Ph.D. thesis, Simon Fraser University, 2014.

[31] A. Morello, J. J. Pla, F. A. Zwanenburg, K. W. Chan, K. Y. Tan, H. Huebl, M. Möttnöen, C. D. Nugroho, C. Yang, J. A. van Donkelaar, A. D. C. Alves, D. N. Jamieson, C. C. Escott, L. C. L. Hollenberg, R. G. Clark, and A. S. Dzurak, Nature (London) 467, 687 (2010).

[32] M. Fuechsle, J. A. Miwa, S. Mahapatra, H. Ryu, S. Lee, O. Warschkow, L. C. L. Hollenberg, G. Klimeck, and M. Y. Simmons, Nat. Nanotechnol. 7, 242 (2012).

[33] C. C. Lo, M. Urdampilleta, P. Ross, M. F. Gonzalez-Zalba, J. Mansir, S. A. Lyon, M. L. W. Thewalt, and J. J. L. Morton, Nat. Mater. 14, 490 (2015).

[34] K. Saeedi, M. Szech, P. Dluhy, J. Z. Salvail, K. J. Morse, H. Riemann, N. V. Abrosimov, N. Notzel, K. L. Litvinenko, B. N. Murdin, and M. L. W. Thewalt, Sci. Rep. 5, 10493 (2015).

[35] S. Lloyd, Science (London) 273, 1073 (1996).

[36] B. Koiller, X. Hu, and S. Das Sarma, Phys. Rev. Lett. 88, 027903 (2001).

[37] M. C. Hersam, N. P. Guisinger, and J. W. Lyding, Nanotechnology 11, 70 (2000).

[38] J. L. O’Brien, S. R. Schofield, M. Y. Simmons, R. G. Clark, A. S. Dzurak, N. J. Curson, B. E. Kane, N. S. McAlpine, M. E. Hawley, and G. W. Brown, Smart Mater. Structures 11, 741 (2002).

[39] M. A. Broome, S. K. Gorman, M. G. House, S. J. Hile, J. G. Keizer, D. Keith, C. D. Hill, T. F. Watson, W. J. Baker, L. C. L. Hollenberg, and M. Y. Simmons, Nat. Commun. 9, 980 (2018).

[40] G. Tosi, F. A. Mohiyaddin, V. Schmitt, S. Tenberg, R. Rahman, G. Klimeck, and A. Morello, Nat. Commun. 8, 450 (2017).

[41] P. T. Greenland, S. A. Lynch, A. F. G. van der Meer, B. N. Murdin, C. R. Pidgeon, B. Redlich, N. Q. Vinh, and G. Aeppli, Nature (London) 465, 1057 (2010).

[42] A. M. Stoneham, Physics 2, 34 (2009).

[43] K. J. Morse, P. Dluhy, J. Huber, J. Z. Salvail, K. Saeedi, H. Riemann, N. V. Abrosimov, P. Becker, H.-J. Pohl, S. Simmons, and M. L. W. Thewalt, Phys. Rev. B 97, 115205 (2018).

[44] K. Stockbridge, S. Chick, E. Crane, A. J. Fisher, and B. N. Murdin (unpublished).

[45] K. R. A. Hazzard, B. Gadway, M. Foss-Feig, B. Yan, S. A. Moses, J. P. Covey, N. Y. Yao, M. D. Lukin, J. Ye, D. S. Jin, and A. M. Rey, Phys. Rev. Lett. 113, 195302 (2014).

[46] N. Q. Vinh, P. T. Greenland, K. Litvinenko, B. Redlich, A. F. G. van der Meer, S. A. Lynch, M. Warner, A. M. Stoneham, G. Aeppli, D. J. Paul, C. R. Pidgeon, and B. N. Murdin, Proc. Natl. Acad. Sci. USA 105, 10649 (2008).

[47] K. Litvinenko, E. Bowyer, P. Greenland, N. Stavrias, J. Li, R. Gwilliam, B. Villis, G. Matmon, M. Pang, B. Redlich,
A. F. G. van der Meer, C. Pidgeon, G. Aeppli, and B. Murdin, Nat. Commun. 6, 6549 (2015).

[48] J. Levy, Phys. Rev. Lett. 89, 147902 (2002).

[49] R. W. Keyes, Computer 38, 65 (2005).

[50] W. Wu and A. J. Fisher, Phys. Rev. B 77, 045201 (2008).

[51] A. K. Ramdas and S. Rodriguez, Rep. Prog. Phys. 44, 1297 (1981).

[52] T. Trappmann, C. Surgers, and H. Lohneysen, Europhys. Lett. 38, 177 (1997).

[53] S. Torquato, B. Lu, and J. Rubinstein, J. Phys. A: Math. Gen. 23, L103 (1990).

[54] D. K. Pickard, J. Appl. Probability 19, 444 (1982).

[55] T. F. Cox, Biometrics 37, 367 (1981).

[56] M. F. Dacey, Geographical Analysis 1, 385 (2010).

[57] T. Monz, K. Kim, W. Hansel, M. Riebe, A. S. Villar, P. Schindler, M. Chwalla, M. Hennrich, and R. Blatt, Phys. Rev. Lett. 102, 040501 (2009).

[58] D. Scott and C. A. Tout, Mon. Not. R. Astron. Soc. 241, 109 (1989).

[59] D. Moltchanov, Ad Hoc Networks 10, 1146 (2012).

[60] J. N. Bahcall and R. M. Soneira, Astrophys. J. 246, 122 (1981).

[61] A. Auerbach, Interacting Electrons and Quantum Magnetism, Graduate Texts in Contemporary Physics (Springer, New York, 2012).

[62] K. Kubo and M. Uchinami, Prog. Theor. Phys. 54, 1289 (1975).

[63] G. Polatsek and K. W. Becker, Phys. Rev. B 54, 1637 (1996).

[64] E. Crane, A. Kolker, T. J. Z. Stock, N. Stavrias, K. Saeedi, M. A. W. van Loon, B. M. Murdin, and N. J. Curson, J. Phys. Conf. Series 1079, 012010 (2018).

[65] A. P. Orioli, A. Signoles, H. Wildhagen, G. Günter, J. Berges, S. Whitlock, and M. Weidemüller, Phys. Rev. Lett. 120, 063601 (2018).

[66] A. Schuckert, A. P. Orioli, and J. Berges, Phys. Rev. B 98, 224304 (2018).

[67] V. Ambegaokar and M. Troyer, Am. J. Phys. 78, 150 (2010).

[68] M. Saffman, T. G. Walker, and K. Mølmer, Rev. Mod. Phys. 82, 2313 (2010).

[69] W. Kohn and J. M. Luttinger, Phys. Rev. 98, 915 (1955).

[70] G. A. Thomas, M. Capizzi, F. DeRosa, R. N. Bhatt, and T. M. Rice, Phys. Rev. B 23, 5472 (1981).

[71] J. Li, N. H. Le, K. L. Litvinenko, S. K. Clowes, H. Engelkamp, S. G. Pavlov, H.-W. Hübers, V. B. Shuman, L. Portsel, A. N. Lodygin, Y. A. Astrov, N. V. Abrosimov, C. R. Pidgeon, A. Fisher, Z. Zeng, Y.-M. Niquet, and B. N. Murdin, Phys. Rev. B 98, 085423 (2018).

[72] T. Hahn, Comput. Phys. Commun. 168, 78 (2005).

[73] E. Crane, Wolfram Demonstrations Project (unpublished).

[74] M. Fewell, Australian Government, Department of Defense Technical Report DSTO-TN-0722 (2006). 